Volume: 2, Issue: 1, January-March 2017, Pages: 127, DOI: http://dx.doi.org/10.19082/ah127

\title{
ASSESSING THE QUALITY OF ELECTRONIC MEDICAL RECORDS IN ACADEMIC HOSPITALS: A MULTICENTER STUDY IN A DEVELOPING COUNTRY
}

\author{
Hedieh Zabolinezhad ${ }^{1}$, Mohammad Reza Hassibian ${ }^{1,2}$, Shahram Amini ${ }^{3}$, Saeid Eslami ${ }^{1,2}$
}

1: Department of Medical Informatics, School of Medicine, Mashhad University of Medical Sciences, Mashhad, Iran

2: Medical Informatics Research Center, School of Medicine, Mashhad, Iran

3: Associate Professor of Anesthesiology And Critical Care, Department of Anesthesiology and Critical Care, Imam Reza Hospital, Mashhad University of Medical Sciences, Mashhad, Iran

Corresponding Author:

Mohammad Reza Hassibian, Department of Medical Informatics, School of Medicine, Mashhad University of Medical Sciences, Mashhad, IR Iran. P. O. Box: 91775-48564. Tel: +98-5138815894, Fax: +98-5138002445, Email: hasibianmr@mums.ac.ir

\section{TYPE OF ARTICLE: CONFERENCE ABSTRACT}

\begin{abstract}
Introduction: The validity of medical research based on electronic databases strongly relies on the quality of recorded data. Although the use of hospital information systems in Iran goes back to 1990s, few studies have assessed the quality of electronic medical records. The aim of this study was to assess the quality of electronic medical records, in the MUMS (Mashhad University of Medical Sciences) hospital information system (HIS), especially valuable ones for education and research.

Methods: Samples of inpatient electronic records were selected in three academic hospitals: one general hospital (A) and two tertiary hospitals (B and C). We categorized all data elements of electronic medical records into five groups, including demographic, identification, diagnosis and treatment, administrative and financial; and laboratory and paraclinic. We asked 25 physicians from three academic hospitals to specify data elements with values of medical research and education (called research and educational data) in every group. Next we calculated recordability, completeness, and accuracy of five data groups according to the concordance between electronic records and corresponding paper records. Quality was calculated as a multiple of completeness and accuracy.

Results: For all data elements, recordability of the software was $58.5 \%$. Quality of demographic, identification, diagnosis and treatment, laboratory and paraclinic, and administrative and financial data groups was $97 \%, 32 \%$, $42 \%, 82 \%, 89 \%$, respectively, in hospital A. Quality of mentioned data groups was $99 \%, 44 \%, 60.5 \%, 91 \%$, and $95.5 \%$ in hospital B, and $98 \%, 41 \%, 61 \%, 30 \%$, and $97 \%$ in hospital C, respectively. For data elements, which were selected as valuable for research and education, recordability of the software was $47 \%$. Also, quality of these data categorized in demographic, identification, diagnosis and treatment, laboratory and paraclinic, and administrative and financial was $100 \%, 67 \%, 48 \%, 89 \%$, and $76 \%$, respectively, in hospital A; $100 \%, 59 \%, 69 \%$, $95 \%$, and $90 \%$ in hospital $\mathrm{B}$; and $100 \%, 34 \%, 65 \%, 32 \%$, and $100 \%$ in hospital C, respectively.
\end{abstract}

Conclusion: The low quality of electronic medical records was a result of incompleteness, while accuracy was relatively good. Results showed that the development and use of hospital information system focused on administrative and financial applications more than academic and clinical applications.

KEYWORDS: Assessing the quality of electronic medical record, Recordability, Completeness, Accuracy, Data quality

\section{Abstracts of First National Congress of Medical Informatics, Mashhad, Iran, February 2017}

(C) 2017 The Authors. This is an open access article under the terms of the Creative Commons Attribution-NonCommercialNoDerivs License, which permits use and distribution in any medium, provided the original work is properly cited, the use is non-commercial and no modifications or adaptations are made. 